\title{
Colocación de catéter ecoguiado para bloqueo PENG continuo en Cirugía de Cadera
}

\section{Catheter placement in continuous PENG blockade in hip surgery}

\author{
Dra. Guadalupe Zaragoza-Lemus, ${ }^{*}$ Dr. Miguel Ángel Céspedes-Korrodi, ${ }^{\ddagger}$ \\ Dra. Daniela Hernández-Rodríguez, ${ }^{\S}$ Dra. Margarita Mancera-Rangel ${ }^{\S}$
}

Citar como: Zaragoza-Lemus G, Céspedes-Korrodi MÁ, Hernández-Rodríguez D, Mancera-Rangel M. Colocación de catéter ecoguiado para bloqueo PENG continuo en Cirugía de Cadera. Rev Mex Anestesiol. 2021; 44 (3): 233-236. https://dx.doi.org/10.35366/99672

\begin{abstract}
RESUMEN. El control de dolor agudo en cirugía de cadera es un reto para el anestesiólogo, no sólo por la complejidad del dolor derivado de las osteotomías en una articulación mayor, sino por la multipatología y fragilidad que acompaña al paciente geriátrico. El bloqueo de los nervios pericapsulares, conocido como «bloqueo PENG» es un bloqueo guiado por ultrasonido puramente sensitivo que inhibe el impulso nociceptivo quirúrgico de tres nervios de la articulación coxofemoral: el nervio femoral, el nervio obturador y el nervio obturador accesorio. Este bloqueo tiene una duración promedio de 10 a 12 horas, prolongar esta eficacia y el margen de seguridad provistos por esta técnica fue el motivo para realizar la colocación de un catéter ecoguiado en el bloqueo PENG en un paciente masculino programado para artroplastía total de cadera con el objetivo de controlar el dolor hasta 48 horas después del procedimiento quirúrgico, optimizar recursos y disminuir su estancia intrahospitalaria y el riesgo de presentación de delirio y efectos adversos derivados del uso de opioides comúnmente asociados en pacientes de edad avanzada. El bloqueo PENG continuo forma parte del manejo multimodal aunado a anestesia general u otras técnicas de anestesia regional, con más horas de analgesia comparado con una dosis única.
\end{abstract}

ABSTRACT. The control of acute pain in hip surgery is a challenge for the anesthesiologist, not only because of the complexity of the pain derived from osteotomies in a larger joint; but because of the multipathology and fragility that accompanies the geriatric patient. The pericapsular nerve block, known as «PENG block» is a purely sensitive ultrasound-guided block that inhibits the surgical nociceptive impulse of three nerves of the hip joint: the femoral nerve, the obturator nerve and the accessory obturator nerve. This block has an average duration of 10 to 12 hours, prolonging this efficiency and the safety margin provided by this technique led us to place an ultrasound-guided catheter in the PENG block for a male patient scheduled for total hip arthroplasty with the objective of controlling pain up to 48 hours after the surgical procedure, optimizing resources and reducing their hospital stay and reducing the risk of delirium and adverse effects derived from the use of opioids commonly associated in elderly patients. Continuous PENG block is part of multimodal management coupled with general anesthesia or other regional anesthesia techniques, with more hours of analgesia compared to a single dose.

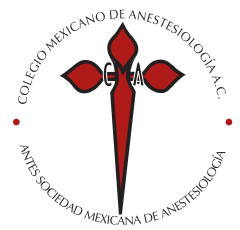

Palabras clave: Cirugía de cadera, analgesia continua, bloqueo PENG, catéter periférico, anestesia regional.

Keywords:

Hip surgery, analgesia continuous, PENG block, peripheral catheter, regional anesthesia.

* Anestesióloga, Algóloga, Anestesia Regional. Instituto Nacional de Rehabilitación, Profesor auxiliar del Curso de Anestesiología.

‡ Residente de 2do año de Anestesiología del Hospital Ángeles Pedregal.

$\S$ Residente de 1er año de Anestesiología del Hospital Ángeles Pedregal.

Universidad La Salle. México.

Correspondencia:

Guadalupe Zaragoza-Lemus Hospital Ángeles Pedregal, Torre de Especialidades Quirúrgicas, Cons. 1176, Camino a Sta. Teresa, Col. Héroes de Padierna, 10700, alcaldía La Magdalena Contreras, Ciudad de México, México. E-mail: zararegional@hotmail.com

Recibido: 25-09-2020 Aceptado:26-01-2021

\section{INTRODUCCIÓN}

L a anestesia regional ecoguiada ha demostrado un gran $\checkmark$ avance en los últimos años para el manejo del dolor perioperatorio en los pacientes sometidos a procedimientos invasivos de cadera. En la actualidad, se utiliza el bloqueo de fascia ilíaca y el bloqueo de nervio femoral para el manejo analgésico en pacientes con fractura de cadera debido a sus beneficios, tales como la disminución del requerimiento de opioides durante el transoperatorio y la reducción en los eventos adversos en sí mismos de los opioides. Recientemente se ha descrito el bloqueo PENG como un bloqueo periférico que ha demostrado superioridad frente a otras técnicas ${ }^{(1)}$.

El bloqueo de los nervios pericapsulares, conocido como «bloqueo PENG», es realizado gracias al estudio anatómico y detallado de la inervación de la articulación coxofemoral descrita en múltiples publicaciones. En el año 2018, Short y colaboradores ${ }^{(2)}$ identificaron la inervación de la cápsula coxofemoral anterior mediante la disección de cadera en 13 cadáveres, describiendo el trayecto del nervio femoral (NF), nervio obturador (NO) y nervio obturador accesorio (NOA). Así como las ramas del NF descienden distalmente para 
inervar la cápsula anterior de la cadera, el NO y NOA aportan ramas nerviosas al plexo nervioso localizado en este componente articular, de manera que el NF se encuentra con mayor facilidad utilizando como referencia la eminencia iliopúbica (EIP) para las ramas superiores y la espina ilíaca anteroinferior (EIAI) para las ramas inferiores; así como el acetábulo inferomedial (AIM) o la «gota de lágrima» para el NO y la EIP para el $\mathrm{NOA}^{(2)}$. Este aporte anatómico fue fundamental para el desarrollo del bloqueo PENG, caracterizado por ser unilateral, específico, selectivo y exclusivamente sensitivo, ya que a diferencia de otras técnicas, este bloqueo tiene efecto en el NOA, no provoca debilidad del músculo cuádriceps como en el bloqueo del nervio femoral y bloqueo 3 en 1; no requiere volúmenes altos como se requiere en el bloqueo de fascia ilíaca y no provoca un bloqueo simpático como en el caso del bloqueo de plexo lumbar ${ }^{(3)}$.

Debido a las ventajas del bloqueo PENG previamente mencionadas, las indicaciones para su uso se pueden ampliar a múltiples escenarios, desde la analgesia para cirugías de cadera o la reducción de luxación coxofemoral, analgesia en fracturas de fémur, hasta prevenir el espasmo de los músculos abductores, analgesia postoperatoria en varicocelectomía, cirugía de glúteo, control del dolor crónico y «on arrival block», entre otros; ya sea como técnica única o como parte del manejo multimodal aunado a anestesia general u otras técnicas de anestesia regional. El objetivo de este reporte fue describir la posibilidad de prolongar la analgesia postoperatoria de 12 a 24 o 48 horas con la colocación de un catéter debajo del tendón del psoas ${ }^{(4)}$.

\section{DESCRIPCIÓN DEL CASO}

Hombre de 51 años sin antecedentes de importancia para el padecimiento actual, alérgico a la penicilina, quien inicia su padecimiento seis meses previos al referir dolor en la articulación coxofemoral izquierda a la marcha, el cual se irradia hacia ingle ipsilateral con EVA 5/10, aumentando de manera progresiva hasta alcanzar EVA 9/10 imposibilitando la deambulación y el sueño. Es valorado por el Servicio de Trauma y Ortopedia, decidiéndose su intervención quirúrgica programándose para artroplastía total de cadera con diagnóstico final de coxartrosis izquierda G-III mediante resonancia magnética. Con un peso de $74 \mathrm{~kg}$ y una talla de $1.77 \mathrm{~m}$. Con exámenes preoperatorios normales: PCR SARS-CoV-2 negativo el día 8 de agosto de 2020, resto de paraclínicos sin alteraciones. EKG normal, Rx tórax sin evidencia de patología. El paciente refiere no ingerir ningún modificador de la coagulación.

\section{Técnica}

Previo consentimiento informado, bajo monitoreo estándar no invasivo, se colocó primero un bloqueo subaracnoideo con $13.5 \mathrm{mg}$ de bupivacaína $0.5 \%$, y posteriormente se colocó para fines de seguridad en la prolongación de la cirugía un catéter epidural inerte con técnica habitual. Se mantuvo con ventilación espontanea $\mathrm{FiO}_{2} 40 \%$, bloqueo motor y sensitivo $100 \%$ al finalizar la cirugía después de 2 horas se colocó en posición decúbito supino, con transductor convexo (Philips C5- $1^{\circledR}$ ) de baja frecuencia (2-5 MHz) en plano transverso sobre la espina ilíaca anteroinferior (EIAI) previa técnica estéril, se alineó con la eminencia iliopúbica (EIP) rotándolo en sentido medial $45^{\circ}$. Durante el escaneo de la sonoanatomía se identificó músculo iliopsoas, tendón del psoas, músculo pectíneo y con Doppler se identificó la arteria femoral (Figuras 1 y 2). Para mayor detalle, consultar la técnica descrita en una publicación de los mismos autores ${ }^{(5)}$. Mediante el equipo Contiplex B. Braun Germany ${ }^{\circledR}$ se introdujo el catéter in plane en dirección de lateral hacia medial (Figura 3), colocando la punta de la aguja $5 \mathrm{~cm}$ por debajo del tendón del psoas observando el plano de hidrodisección, verificando en todo momento la

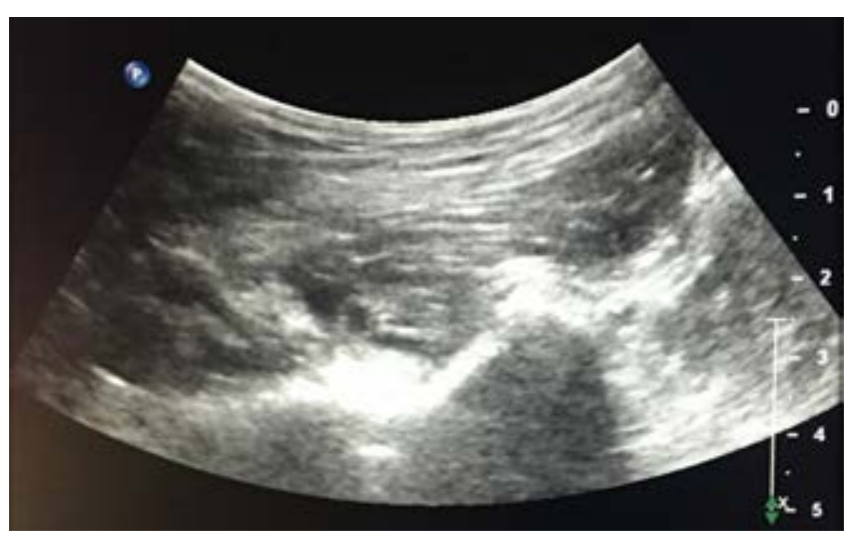

Figura 1: Sonoanatomía del bloqueo PENG.

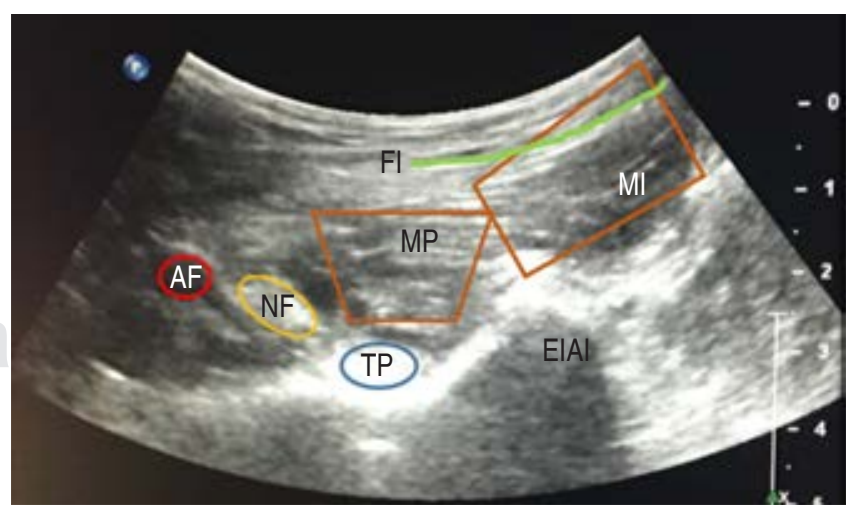

Figura 2: Sonoanatomía.

$\mathrm{FI}=$ Fascia ilíaca, $\mathrm{MI}=$ músculo ilíaco, $\mathrm{MP}=$ músculo psoas, $\mathrm{NF}=$ nervio femoral, $\mathrm{AF}=$ arteria femoral, $\mathrm{TP}=$ tendón del psoas, $\mathrm{EIAI}=$ espina ilíaca anteroinferior. 


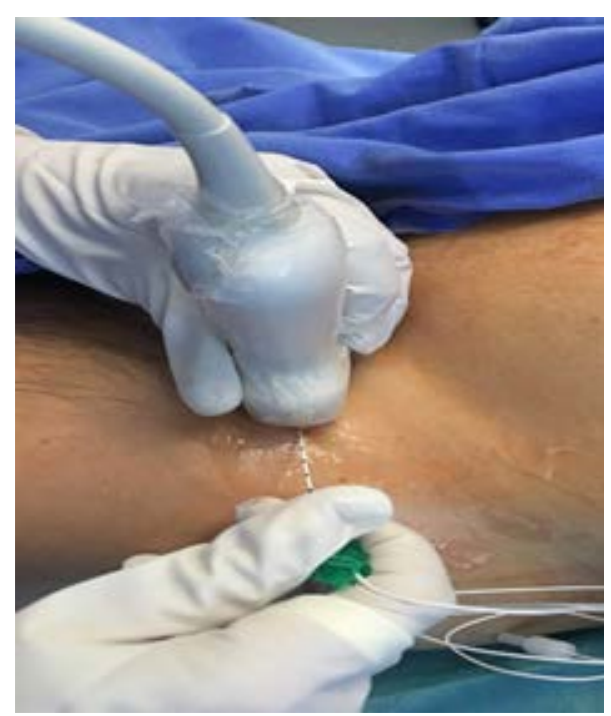

Figura 3: Inserción de la aguja Contiplex in plane.

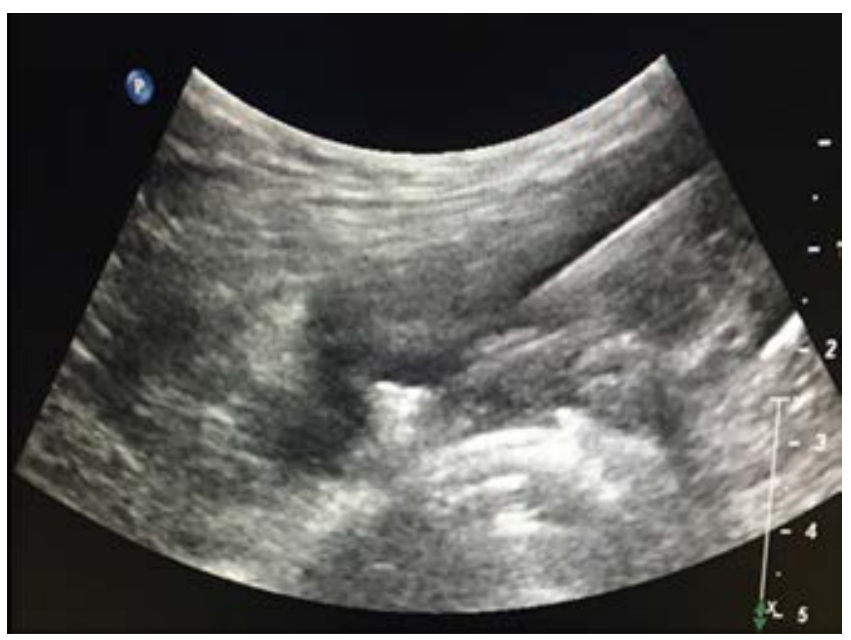

Figura 4: Inserción de la aguja al tendón del psoas.

arteria femoral para evitar punción vascular (Figura 4). Se realizó aspiración de la jeringa y se depositó el anestésico local (LD dosis de caga): $20 \mathrm{~mL}$ de ropivacaína $0.25 \%$, observando la elevación del tendón del músculo psoas. Se fijó a la piel y se colocó bomba HomePump ${ }^{\circledR}$ a una tasa 5 $\mathrm{mL} / \mathrm{h}$ con un volumen total de $120 \mathrm{~mL}$ con ropivacaína al $2.0 \%$ para cada 24 horas (Figuras 5 y 6 ).

\section{Evolución y seguimiento}

Durante el postoperatorio se registró la escala visual análoga (EVA) 30 minutos después del bloqueo, así como cada 2 horas obteniendo en promedio, un puntaje de 2 a 3/10 de EVA en movilización pasiva flexionando la cadera $30^{\circ}$, no se administraron rescates analgésicos al esquema habitual. El paciente continuó refiriendo EVA 3/10 a las 24 horas aun al iniciar con movimientos activos para dar comienzo a la marcha, se midió la fuerza muscular $5 / 5$. Se retira catéter 48 horas después del bloqueo sin complicaciones. Fue egresado del hospital sin ningún incidente, se realizó una encuesta de satisfacción a la cual respondió con el máximo puntaje.

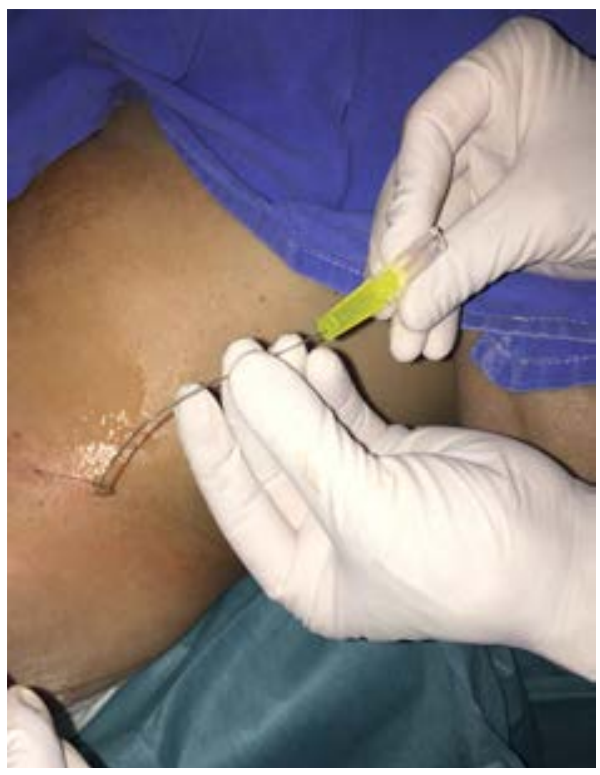

Figura 5: Catéter a la piel.

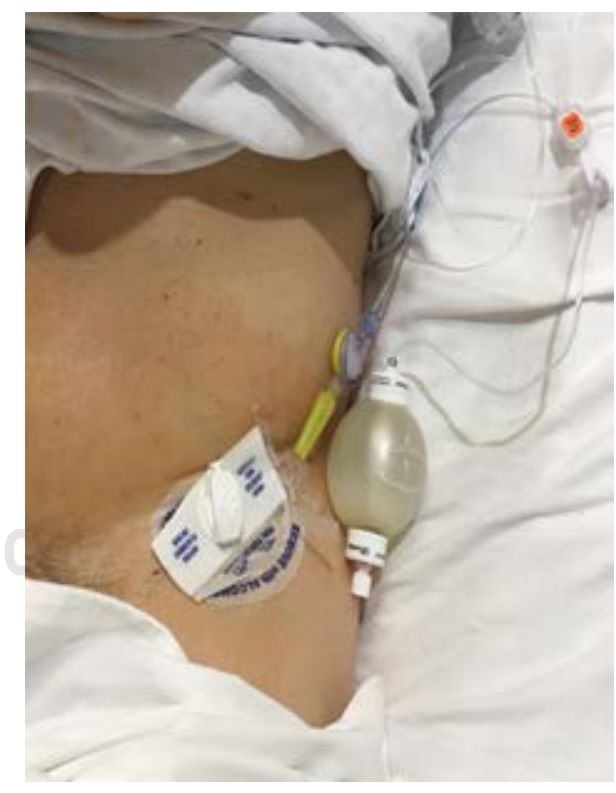

Figura 6: Fijación del catéter y bomba elastomérica. 


\section{CONCLUSIONES Y DISCUSIÓN}

Mediante el control analgésico logrado bajo esta técnica, comenzó a movilizar el miembro afectado dos horas después de la cirugía, poniéndose de pie a las 24 horas, facilitando el inicio de la marcha y su recuperación disminuyendo la estancia intrahospitalaria. No se presentaron complicaciones clínicas o técnicas como hematoma ni dislocación o disfunción del catéter. La colocación de catéteres por ultrasonido corresponde a una técnica avanzada, por lo que requiere una formación clínica con entrenamiento, para que no varíen los resultados finales. Los autores recomiendan hacer la entrada de la aguja Contiplex superior a 90 grados y reportamos que, dada la maleabilidad de esta aguja y el pequeño calibre del catéter que no es ecolúcido, se debe aumentar la precaución en el momento de inserción ${ }^{(5)}$. A diferencia de otros autores, el catéter se colocó medial al tendón del psoas una vez que pasó justo por debajo de éste para mejorar la estabilidad del catéter, ya que avanzó dentro de los músculos psoas e ilíaco ${ }^{(6,7)}$. Al tratar de instalar la técnica, los autores ensayaron y recomiendan el uso de la aguja de Touhy G-17 cuando no se disponga del equipo Contiplex y por último se detectó que es mandatorio el uso de transductor de baja frecuencia en la colocación de catéter ecoguiado PENG, no se recomienda el uso del transductor lineal o de alta frecuencia ${ }^{(8)}$.

\section{REFERENCIAS}

1. Girón-Arango L, Peng PWH, Chin KJ, Brull R, Perlas A. Pericapsular nerve group (PENG) block for hip fracture. Reg Anesth Pain Med. 2018;43:859-863.

2. Short AJ, Barnett JJG, Gofeld M, Baig E, Lam K, Agur AMR et al. Anatomic study of innervation of the anterior hip capsule: implication for image-guided intervention. Reg Anesth Pain Med. 2018;43:186-192.

3. Guay J, Parker MJ, Griffiths R, Kopp S. Peripheral nerve blocks for hip fractures. Cochrane Database Syst Rev. 2017;5:CD001159.

4. Suksompong S, von Bormann S, von Bormann B. Regional catheters for postoperative pain control: review and observational data. Anesth Pain Med. 2020;10:e99745.
5. Zaragoza-Lemus G, Portela-Ortiz JM, Díaz-Guevara G. Bloqueo del grupo de nervios pericapsulares (PENG) para cirugía de cadera. Rev Mex Anest. 2020;43:69-72.

6. Avers D, Brown M. Daniels and Worthingham's muscle testing. Techniques of manual examination and performance testing. St Louis: Elsevier; 2018.

7. Del Buono R, Padua E, Pascarella G, Soare CG, Barbara E. Continuous PENG block for hip fracture: a case series. Reg Anesth Pain Med. 2020;45:835-838. doi: 10.1136/rapm-2020-101446.

8. Santos O, Pereira R, Cabral T, Lages N, Machado H. Is continuous PENG block the new 3-in-1 J Anesth Clin Res. 2019;10:898. 